\title{
Effects of Catalyst Activation and Ligand Steric Properties on the Enantioselective Allylation of Amines and Phenoxides
}

\author{
Supporting Information
}

\author{
Andreas Leitner, Chutian Shu and John F. Hartwig* \\ Yale University, Department of Chemistry \\ P.O. Box 208107, New Haven, Connecticut 06520-8107 \\ John.Hartwig@yale.edu
}


General: All manipulations were carried out under an inert atmosphere using a nitrogenfilled glovebox or standard Schlenk techniques. All glassware was oven or flame dried immediately prior to use. THF was obtained as HPLC grade without inhibitors; benzene, toluene, dichloromethane, and pentane were obtained as ACS reagent grade. THF, diethyl ether, benzene, toluene, dichloromethane, and pentane were degassed by purging with nitrogen for 45 min and dried with a solvent purification system containing a $1 \mathrm{~m}$ column containing activated alumina. All reagents were obtained from commercial sources and used without further purification. ${ }^{1} \mathrm{H}$ NMR spectra were obtained at 400 or $500-\mathrm{MHz}$ and recorded relative to residual protio-solvent. ${ }^{13} \mathrm{C}$ NMR spectra were obtained at 101 or $126 \mathrm{MHz}$, and chemical shifts were recorded relative to the solvent resonance. $[\operatorname{Ir}(\mathrm{COD}) \mathrm{Cl}]_{2}, \mathrm{O}, \mathrm{O}^{\prime}-(R)-\left(1,1^{\prime}-\mathrm{Dinaphthyl}-2,2^{\prime}-\right.$ diyl $)-\mathrm{N}, \mathrm{N}^{\prime}-\mathrm{di}-(R, R)-$ phenylethylphosphoramidite, ${ }^{1} \quad$ and $\quad \mathrm{O}, \mathrm{O}^{\prime}-(R)-\left(1,1^{\prime}-\mathrm{Dinaphthyl}-2,2^{\prime}-\operatorname{diyl}\right)-\mathrm{N}, \mathrm{N}^{\prime}-\mathrm{di}-(R, R)-$ naphthylethylphosphoramidite ${ }^{2}$ were prepared according to published procedures. All allylic carbonates were prepared by the reaction of the corresponding allylic alcohol with methyl chloroformate in the presence of pyridine. (E)-4-methoxycinnamyl alcohol, (E)-2methoxycinnamyl alcohol, (E)-3-(2-furanyl)-2-propen-1-ol, and (E)-4-methyl-2-penten-1-ol were prepared by reduction of the corresponding aldehyde with $\mathrm{NaBH}_{4} / \mathrm{CeCl}_{3}$.

\section{General Procedures for the Enantioselective Allylic Amination.}

Method A: Catalyst activation with propylamine. $[\operatorname{Ir}(\mathrm{COD}) \mathrm{Cl}]_{2} \quad(0.070 \mathrm{mmol})$, phosphoramidite ligand ( $\mathbf{L 1}$ or $\mathbf{L 2})(0.140 \mathrm{mmol})$ were diluted in $0.3 \mathrm{~mL}$ of THF and $0.3 \mathrm{~mL}$ propylamine and heated at $50{ }^{\circ} \mathrm{C}$ for $20 \mathrm{~min}$. After this time, all volatile materials were evaporated. The yellow solid was diluted in $3.5 \mathrm{~mL}$ of THF to generate a stock solution of the activated catalyst. In a drybox, amine $(1.20 \mathrm{mmol})$ was added to $0.5 \mathrm{~mL}$ of the stock solution of the catalyst $(0.020 \mathrm{mmol})$ in a screw capped vial containing a stirring bar. The vial was sealed with a cap containing a PTFE septum and removed from the drybox. Carbonate (1.00 mmol) was added with a syringe, and the reaction was stirred at room temperature until the carbonate was fully converted, as determined by GC and TLC. The volatile materials were evaporated. The ratio of regioisomers (branched to linear b/l) was determined by ${ }^{1} \mathrm{H}$ NMR or gas chromatographic analysis of the crude mixture. The crude reaction mixture was purified by flash column chromatography on silica gel (hexanes/ether) to give desired product. 
Method B: Catalyst activation with DABCO. In a drybox, DABCO (0.100 mmol), $[\operatorname{Ir}(\mathrm{COD}) \mathrm{Cl}]_{2}(0.010 \mathrm{mmol})$ and phosphoramidite ligand $(\mathbf{L 1}$ or L2) $(0.020 \mathrm{mmol})$ were dissolved in $0.5 \mathrm{~mL}$ of THF in a screw-capped vial. A small magnetic stirbar was added, and the vial was sealed with a cap containing a PTFE septum. The vial was removed from the drybox. Amine $(1.20 \mathrm{mmol})$ and methyl carbonate $(1.00 \mathrm{mmol})$ were added by syringe, and the reaction was stirred at room temperature until the carbonate was fully converted, as determined by GC and TLC. The volatile materials were evaporated. The ratio of regioisomers (branched to linear b/l) was determined by ${ }^{1} \mathrm{H}$ NMR or gas chromatographic analysis of the crude mixture. The crude reaction mixture then was purified by flash column chromatography on silica gel (hexanes/ether) to give desired product.

\section{Allylic Alkylation conducted with $0, O^{\prime}-(R)-\left(1,1^{\prime}-D i n a p h t h y l-2,2^{\prime}-d i y l\right)-N, N^{\prime}-d i-(R, R)-$ naphthylethylphosphoramidite (L2):}

$N$-[1-(n-Propyl)-2-propenyl]benzylamine. ${ }^{3}$ The general procedure of method A was followed with methyl carbonate derived from $(E)$-2-hexen-1-ol $(0.152 \mathrm{~g}, 0.94 \mathrm{mmol})$ and benzylamine $(0.140 \mathrm{~mL}, 1.28 \mathrm{mmol})$. The reaction was conducted at room temperature for $0.5 \mathrm{~h}$. ${ }^{1} \mathrm{H}$ NMR analysis of the crude reaction mixture indicated the ratio of regioisomers (b/l) to be 94/6. The mixture was purified by flash column chromatography on silica gel (hexanes/ether 10:1 to $5: 1)$ to give the title compound $(0.122 \mathrm{~g}, 67 \%)$. HPLC analysis indicated that the enantiomeric excess of the product was 98\% [Diacel CHIRALCEL OD-H $(0.46 \mathrm{~cm}$ x $25 \mathrm{~cm})$; hexanes $/ 2$-propanol $=98.0 / 2.0 ;$ flow rate $=0.5 \mathrm{~mL} / \mathrm{min}$; detection wavelength $=230 \mathrm{~nm} ; \mathrm{TR}=$ 11.2 (major), 9.10 (minor) min]. ${ }^{1} \mathrm{H}$ NMR (500.13 MHz, $\left.\mathrm{CDCl}_{3}\right) \delta=7.22-7.32$ (m, 5H), 5.62 (ddd, $J=16.8,10.4,7.6 \mathrm{~Hz}, 1 \mathrm{H}), 5.14$ (dd, $J=10.4,1.6 \mathrm{~Hz}, 1 \mathrm{H}), 5.10$ (dd, $J=16.8,1.6 \mathrm{~Hz}, 1 \mathrm{H})$, $3.83(\mathrm{~d}, J=13.2 \mathrm{~Hz}, 1 \mathrm{H}), 3.64$, (d, $J=13.2 \mathrm{~Hz}, 1 \mathrm{H}), 3.03(\mathrm{dt}, J=7.6,6.0 \mathrm{~Hz}, 1 \mathrm{H}), 1.25-1.52$ $(\mathrm{m}, 5 \mathrm{H}), 0.89(\mathrm{t}, J=7.2 \mathrm{~Hz}, 3 \mathrm{H}) .{ }^{13} \mathrm{C} \mathrm{NMR}\left(125.7 \mathrm{MHz}, \mathrm{CDCl}_{3}\right) \delta=141.3,140.7,128.3,128.1$, 126.7, 115.9, 61.0, 51.2, 37.9, 19.1, 14.0.

$\boldsymbol{N}$-Phenyl-1-n-propyl-2-propenylamine. ${ }^{3}$ The general procedure was followed with the methyl carbonate derived from $(E)$-2-hexen-1-ol (0.149 g, $0.94 \mathrm{mmol})$ and aniline (109 mL, 1.20 mmol). ${ }^{1} \mathrm{H}$ NMR analysis of the residue crude mixture indicated that the ratio of regioisomers (b/l) was 92/8. The crude reaction mixture was purified by flash column chromatography on silica gel (hexanes/ether 20:1) to give desired product (0.161 g, 93\%) as colourless liquid. HPLC 
analysis indicated that the enantiomeric excess of the product was 92\% [Diacel CHIRALCEL OD-H $(0.46 \mathrm{~cm}$ x $25 \mathrm{~cm})$; hexanes $/ 2$-propanol $=99.95 / 0.05$; flow rate $=0.5 \mathrm{~mL} / \mathrm{min}$; detection wavelength $=254 \mathrm{~nm}$; TR $=14.3$ (major), 18.3 (minor) $\min ] .[\alpha]_{\mathrm{D}}{ }^{25}=-8.30\left(c 0.995, \mathrm{CHCl}_{3}\right)$. ${ }^{1} \mathrm{H}$ NMR $\left(400.13 \mathrm{MHz}, \mathrm{CDCl}_{3}\right) \delta=7.22-7.14(\mathrm{~m}, 2 \mathrm{H}), 6.71(\mathrm{t}, J=7.4 \mathrm{~Hz}, 1 \mathrm{H}), 6.63(\mathrm{dd}, J=8.0$, $0.8 \mathrm{~Hz}, 2 \mathrm{H}), 5.77$ (ddd, $J=17.2,10.4,6.0 \mathrm{~Hz}, 1 \mathrm{H}), 5.24$ (d, $J=17.2 \mathrm{~Hz}, 1 \mathrm{H}), 5.15$ (d, $J=10.2$ Hz, 1 H), 3.85 (q, $J=6.4$ Hz, 1H), 3.65 (br s, 1 H), 1.65-1.55 (m, 2H), 1.53-1.43 (m, 2H), 0.99 $(\mathrm{d}, J=7.4 \mathrm{~Hz}, 3 \mathrm{H}) .{ }^{13} \mathrm{C} \mathrm{NMR}\left(100.59 \mathrm{MHz}, \mathrm{CDCl}_{3}\right) \delta=147.6,140.1,129.1,117.0,114.9$, $113.2,55.6,38.0,19.1,14.0$.

$N$-(furan-2-ylmethyl)hex-1-en-3-amine. The general procedure of method A was followed with the methyl carbonate derived from $(E)$-2-hexen-1-ol $(0.157 \mathrm{~g}, 0.99 \mathrm{mmol})$ and furfurylamine $(0.111 \mathrm{~mL}, 1.20 \mathrm{mmol})$. The reaction was conducted at room temperature for $1 \mathrm{~h} .{ }^{1} \mathrm{H}$ NMR analysis of the crude reaction mixture indicated the ratio of regioisomers (b/l) to be $94 / 6$. The mixture was purified by flash column chromatography on silica gel (hexanes/ether 10/1 to 5/1) to give the title compound $(0.138 \mathrm{~g}, 85 \%)$. HPLC analysis indicated that the enantiomeric excess of the product was 98\% [Diacel CHIRALCEL OD-H $(0.46 \mathrm{~cm} \times 25 \mathrm{~cm})$; hexanes/2-propanol= 99/1.0; flow rate $=0.5 \mathrm{~mL} / \mathrm{min}$; detection wavelength $=254 \mathrm{~nm}$; $\mathrm{TR}=10.2$ (major), 7.04 (minor) $\min ] .[\alpha]_{\mathrm{D}}{ }^{25}=+26.7\left(c 1.050, \mathrm{CHCl}_{3}\right) .{ }^{1} \mathrm{H} \mathrm{NMR}\left(\mathrm{CDCl}_{3}, 500.13 \mathrm{MHz}\right) \delta=7.40(\mathrm{~m}, 1 \mathrm{H}), 6.37$ (m, 1H), $6.17(\mathrm{~m}, 1 \mathrm{H}), 5.61(\mathrm{~m}, 1 \mathrm{H}), 5.18(\mathrm{~m}, 2 \mathrm{H}), 3.86(\mathrm{~d}, J=14.6 \mathrm{~Hz}, 1 \mathrm{H}), 3.71(\mathrm{~d}, J=14.3$ $\mathrm{Hz}, 1 \mathrm{H}), 3.05(\mathrm{~m}, 1 \mathrm{H}), 1.58-1.30(\mathrm{~m}, 5 \mathrm{H}), 0.90(\mathrm{t}, J=7.2 \mathrm{~Hz}, 3 \mathrm{H}) .{ }^{13} \mathrm{C} \mathrm{NMR}(125.7 \mathrm{MHz}$, $\left.\mathrm{CDCl}_{3}\right) \delta=154.0,141.6,140.9,116.4,110.0,106.7,60.7,43.5,37.7,19.0,14.0$. 


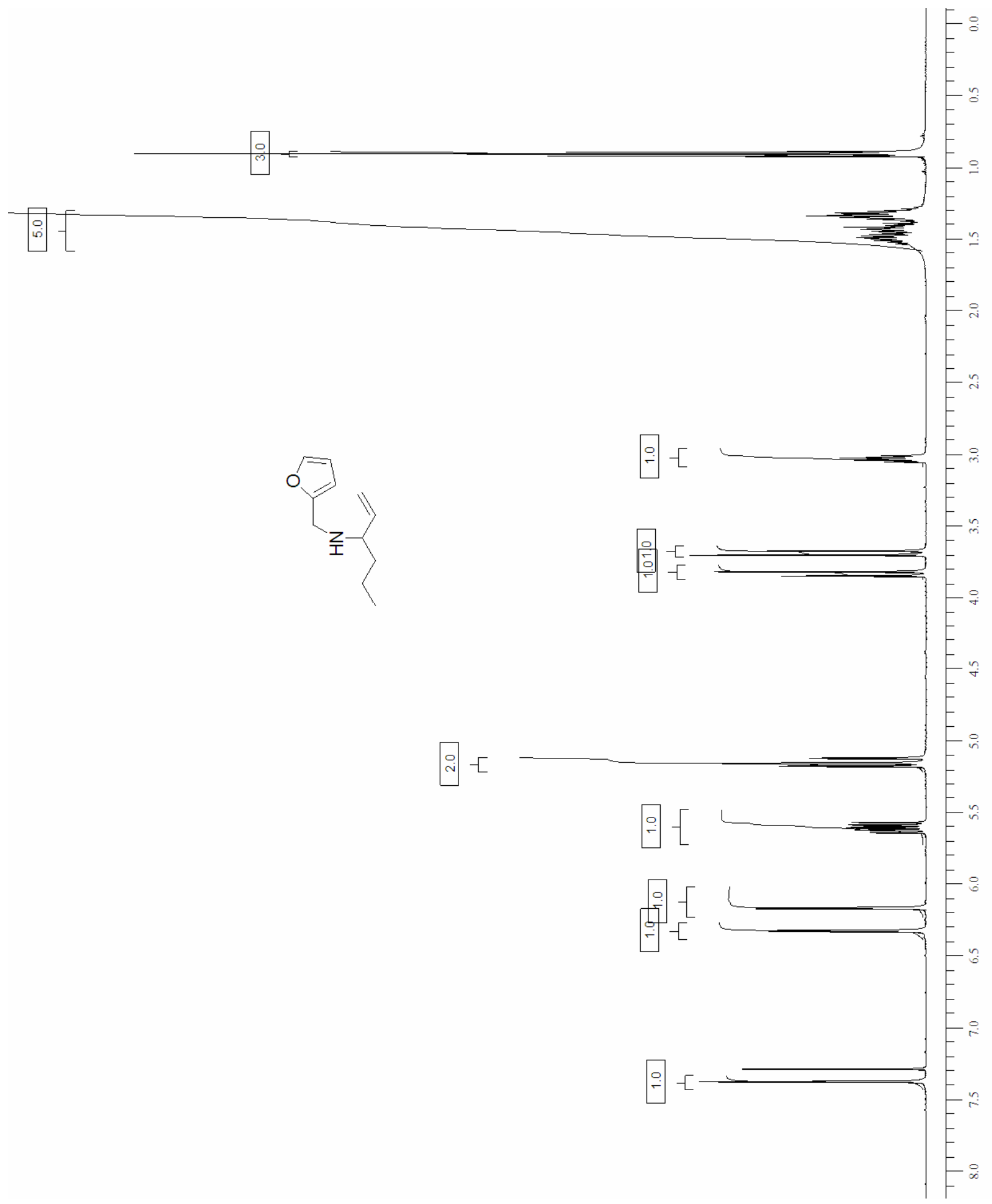


$t \angle 6^{\circ} \mathcal{E} \mathrm{I}$

$\varsigma 00{ }^{\circ} 6 \mathrm{I}$

$\varepsilon Z L \cdot L \mathcal{E}$

$9 t \varsigma^{*} \varepsilon t$

$£ 99^{\circ} 09$

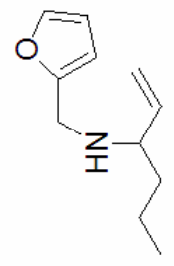

$689^{\circ} 90$ I

$066^{\circ} 60$ I

IEt*9 I I-

$6 \subseteq 8^{\circ} 0 t[$

tt9. It I

I $t 0^{\circ} t \varsigma \mathrm{I}$

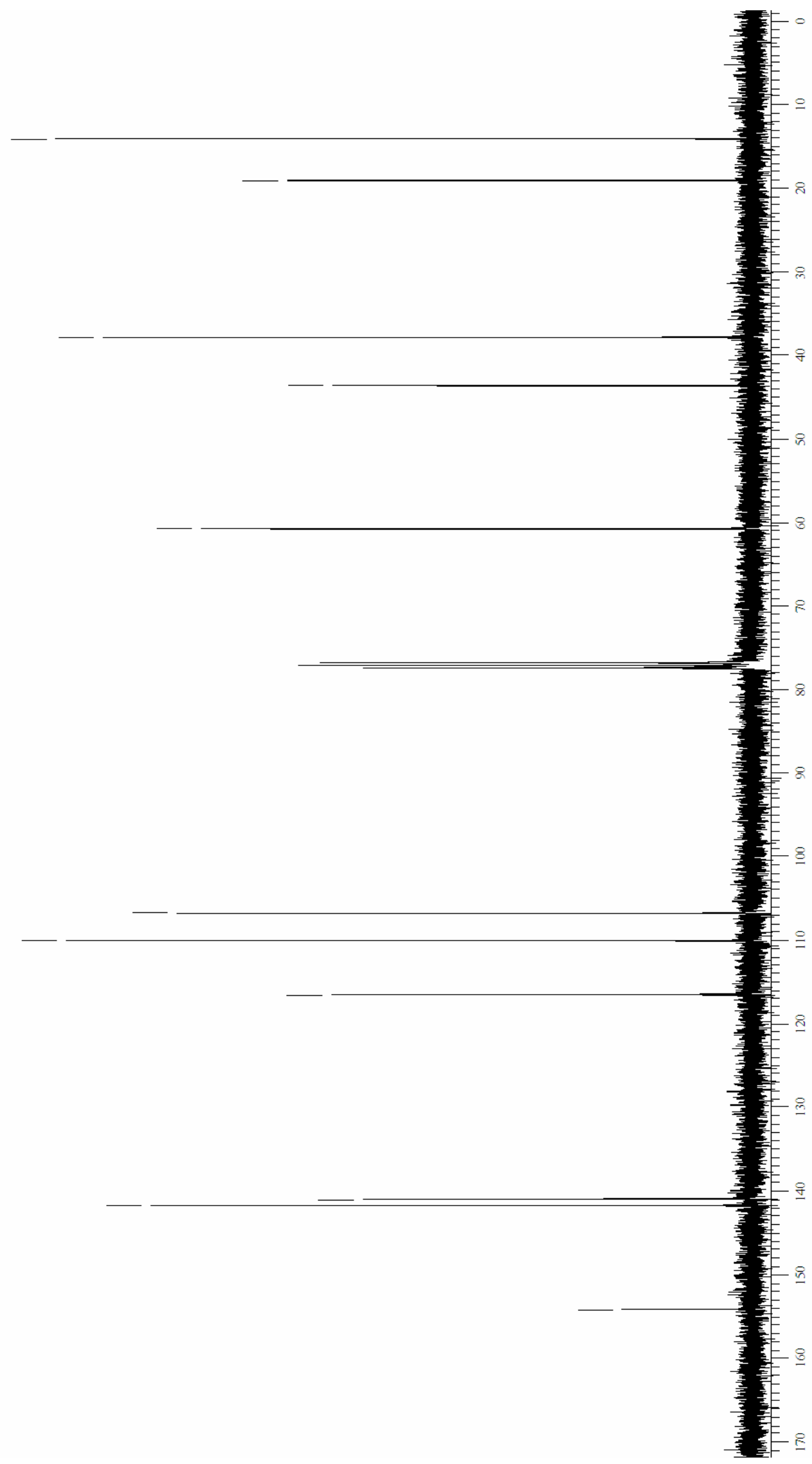


$N$-(thiophen-2-ylmethyl)hex-1-en-3-amine. The general procedure of method A was followed with the methyl carbonate derived from $(E)$-2-hexen-1-ol $(0.147 \mathrm{~g}, 0.95 \mathrm{mmol})$ and 2thiophenemethanamine $(0.138 \mathrm{~mL}, 1.30 \mathrm{mmol})$. The reaction was conducted at room temperature for $1 \mathrm{~h} .{ }^{1} \mathrm{H}$ NMR analysis of the crude reaction mixture indicated the ratio of regioisomers (b/l) to be 93/7. The mixture was purified by flash column chromatography on silica gel (hexanes/ether $10 / 1$ to $5 / 1)$ to give the title compound $(0.144 \mathrm{~g}, 86 \%)$. HPLC analysis indicated that the enantiomeric excess of the product was 98\% [Diacel CHIRALCEL OD-H $(0.46 \mathrm{~cm} \times 25 \mathrm{~cm})$; hexanes $/ 2$-propanol $=99.0 / 1.0$; flow rate $=0.5 \mathrm{~mL} / \mathrm{min}$; detection wavelength $=230 \mathrm{~nm}$; TR $=11.6$ (minor), 10.6 (major) $\min ] .[\alpha]_{\mathrm{D}}{ }^{25}=+14.8\left(c 1.39, \mathrm{CHCl}_{3}\right) .{ }^{1} \mathrm{H}$ $\operatorname{NMR}\left(500.13 \mathrm{MHz}, \mathrm{CDCl}_{3}\right) \delta=7.22(\mathrm{dd}, J=5.1,1.1 \mathrm{~Hz}, 1 \mathrm{H}), 6.94(\mathrm{~m}, 1 \mathrm{H}), 6.92(\mathrm{~m}, 1 \mathrm{H}), 5.60$ (m, 1H), $5.14(\mathrm{~m}, 2 \mathrm{H}), 4.05(\mathrm{~d}, J=14.2 \mathrm{~Hz}, 1 \mathrm{H}), 3.87(\mathrm{~d}, J=14.2 \mathrm{~Hz}, 1 \mathrm{H}), 3.06(\mathrm{~m}, 1 \mathrm{H}), 1.52-$ $1.27(\mathrm{~m}, 4 \mathrm{H}), 0.89$ (t, $J=7.3 \mathrm{~Hz}, 3 \mathrm{H}) .{ }^{13} \mathrm{C} \mathrm{NMR}\left(125.7 \mathrm{MHz}, \mathrm{CDCl}_{3}\right) \delta=144.6,141.0,126.5$, 124.6, 124.1, 116.2, 60.5, 45.6, 37.9, 19.0, 14.0. 


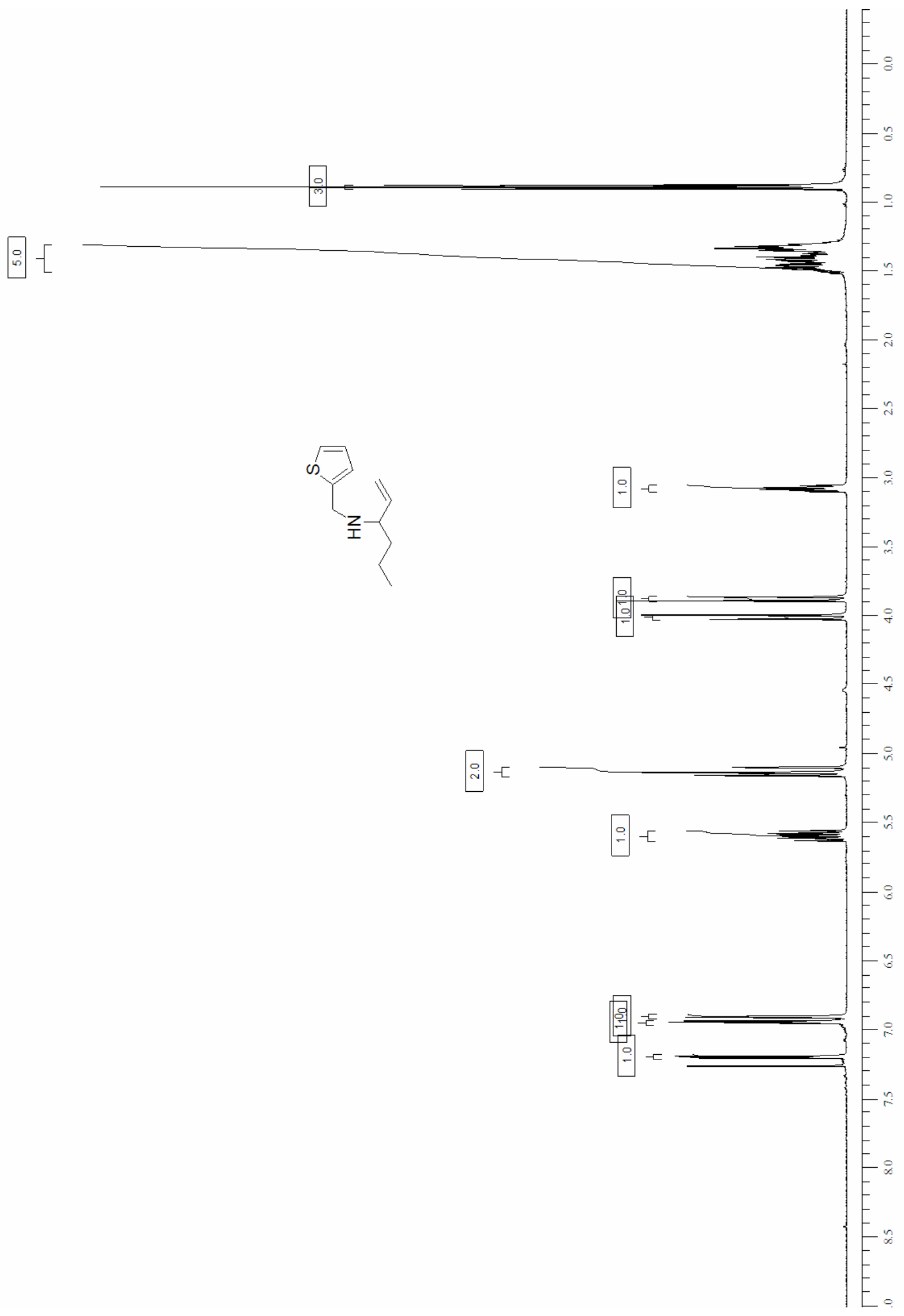


$\varepsilon z 0^{\circ} t$

$\angle t 00^{\circ} \mathrm{I}$

$\angle \varsigma 8^{\circ} L \mathcal{E}$

$88 \varsigma^{\prime} \mathrm{st}$

0ะऽ 09

89I'9I L

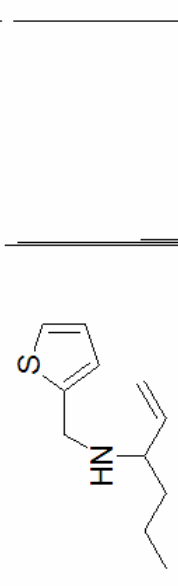

soI' $t z I m$

$\varepsilon 6 \varsigma^{\circ}+z \mathrm{I}$

SIS" $9 Z 1-$

t86 0 t

t8s.tt I

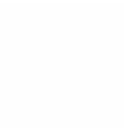


$\mathrm{N}$-(hex-1-en-3-yl)-4-(methylthio)benzenamine. The general procedure of method A was followed with the methyl carbonate derived from $(E)$-2-hexen-1-ol (0.148 g, $0.93 \mathrm{mmol})$ and 4aminothioanisole $(0.146 \mathrm{~mL}, 1.20 \mathrm{mmol})$. The reaction was conducted at room temperature for 1 h. ${ }^{1} \mathrm{H}$ NMR analysis of the crude reaction mixture indicated the ratio of regioisomers (b/l) to be $>99 / 1$. The mixture was purified by flash column chromatography on silica gel (hexanes/ether $10 / 1)$ to give the title compound $(0.198 \mathrm{~g}, 92 \%)$. HPLC analysis indicated that the enantiomeric excess of the product was 98\% [Diacel CHIRALCEL OD-H $(0.46 \mathrm{~cm} \times 25 \mathrm{~cm})$; hexanes/2propanol $=99.5 / 0.5 ;$ flow rate $=0.5 \mathrm{~mL} / \mathrm{min}$; detection wavelength $=230 \mathrm{~nm} ; \mathrm{TR}=18.1$ (major), 19.0 (minor) min]. $[\alpha]_{\mathrm{D}}{ }^{25}=-4.1\left(c 0.82, \mathrm{CHCl}_{3}\right) .{ }^{1} \mathrm{H} \mathrm{NMR}\left(500.13 \mathrm{MHz}, \mathrm{CDCl}_{3}\right) \delta=$ $7.19(\mathrm{~d}, J=8.5 \mathrm{~Hz}, 2 \mathrm{H}), 6.55(\mathrm{~d}, J=8.6 \mathrm{~Hz}, 2 \mathrm{H}), 5.72(\mathrm{ddd}, J=16.9,10.5,6.4 \mathrm{~Hz}, 1 \mathrm{H}), 5.19$ $(\mathrm{dt}, J=16.9,1.4 \mathrm{~Hz}, 1 \mathrm{H}), 5.12(\mathrm{dt}, J=10.3,1.1 \mathrm{~Hz}, 1 \mathrm{H}), 3.78(\mathrm{~m}, 2 \mathrm{H}), 2.40(\mathrm{~s}, 3 \mathrm{H}), 1.60-1.54$ $(\mathrm{m}, 2 \mathrm{H}), 1.46-1.38(\mathrm{~m}, 2 \mathrm{H}), 0.94(\mathrm{t}, J=7.6 \mathrm{~Hz}, 3 \mathrm{H}) .{ }^{13} \mathrm{C} \mathrm{NMR}\left(125.7 \mathrm{MHz}, \mathrm{CDCl}_{3}\right) \delta=146.4$, 139.8, 131.5, 123.9, 115.1, 113.9, 55.8, 37.9, 19.2, 19.1, 13.9. Anal. Calcd. for $\mathrm{C}_{13} \mathrm{H}_{19} \mathrm{NS}_{\mathrm{N}} \mathrm{C}$, 70.54; H, 8.65; N, 6.33; S, 14.49. Found: C, 70.34; H, 9.26; N, 6.30.

(E)- $N$-(furan-2-ylmethyl)hexa-1,4-dien-3-amine. The general procedure of method B was followed with methyl $(2 E, 4 E)$-hexa-2,4-dienylcarbonate $(0.149 \mathrm{~g}, 0.95 \mathrm{mmol})$, furfurylamine (0.140 mL, $1.58 \mathrm{mmol}),[\operatorname{Ir}(\mathrm{COD}) \mathrm{Cl}]_{2}(6.7 \mathrm{mg}, 0.010 \mathrm{mmol}), \mathbf{L 2}(12.7 \mathrm{mg}, 0.02 \mathrm{mmol})$ and DABCO (10 mg, $0.09 \mathrm{mmol})$. The reaction was conducted at room temperature for $1 \mathrm{~h}$. GC analysis of the crude reaction mixture indicated the ratio of regioisomers (b/l) to be $91 / 9$. The mixture was purified by flash column chromatography on silica gel (hexanes/ether 10/1 to 5/1) to give the title compound $(0.128 \mathrm{~g}, 76 \%)$. HPLC analysis indicated that the enantiomeric excess of the product was 97\% [Diacel CHIRALCEL OD-H $(0.46 \mathrm{~cm}$ x $25 \mathrm{~cm})$; hexanes/2-propanol = 99.9/0.1; flow rate $=0.5 \mathrm{~mL} / \mathrm{min}$; detection wavelength $=230 \mathrm{~nm}$; TR $=8.72$ (major), 7.85 (minor) $\min ] .[\alpha]_{\mathrm{D}}{ }^{25}=+13.2\left(c 1.42, \mathrm{CHCl}_{3}\right) .{ }^{1} \mathrm{H} \mathrm{NMR}\left(500.13 \mathrm{MHz}, \mathrm{CDCl}_{3}\right) \delta=7.35(\mathrm{~m}, 1 \mathrm{H})$, $6.30(\mathrm{~m}, 1 \mathrm{H}), 6.15(\mathrm{~m}, 1 \mathrm{H}), 5.76(\mathrm{ddd}, J=17.3,10.3,7.2 \mathrm{~Hz}, 1 \mathrm{H}), 5.61(\mathrm{dq}, J=15.2,6.6,1 \mathrm{H})$, 5.39 (ddq, $J=15.2,7.6,1.6 \mathrm{~Hz}, 1 \mathrm{H}), 5.16$ (dt, $J=16.6,1.6 \mathrm{~Hz}, 1 \mathrm{H}), 5.10(\mathrm{dm}, J=10.0 \mathrm{~Hz}, 1 \mathrm{H})$, $3.74(\mathrm{~s}, 2 \mathrm{H}), 3.59$ (t, $J=7.4 \mathrm{~Hz}, 1 \mathrm{H}), 1.70(\mathrm{dd}, J=6.7,1.6 \mathrm{~Hz}, 3 \mathrm{H}), 1.65-1.50$ (br s, $1 \mathrm{H}) .{ }^{13} \mathrm{C}$ NMR $\left(125.7 \mathrm{MHz}, \mathrm{CDCl}_{3}\right) \delta=153.9,141.7,139.8,132.1,127.3,115.5,110.0,106.8,62.7,43.3$, 
17.8. Anal. Calcd. for $\mathrm{C}_{11} \mathrm{H}_{15} \mathrm{NO}$ : C, 74.54; H, 8.53; N, 7.90; O, 9.03. Found: C, 74.29; H, 8.76; N, 8.01.

(E)-N-(thiophen-2-ylmethyl)hexa-1,4-dien-3-amine. The general procedure of method B was followed with methyl $(2 E, 4 E)$-hexa-2,4-dienylcarbonate $(0.157 \mathrm{~g}, 1.00 \mathrm{mmol})$, 2thiophenemethanamine $(0.138 \mathrm{~mL}, 1.35 \mathrm{mmol}),[\operatorname{Ir}(\mathrm{COD}) \mathrm{Cl}]_{2}(6.7 \mathrm{mg}, 0.010 \mathrm{mmol}), \mathbf{L 2}(12.7$ $\mathrm{mg}, 0.02 \mathrm{mmol})$ and DABCO $(10 \mathrm{mg}, 0.09 \mathrm{mmol})$. The reaction was conducted at room temperature for $2 \mathrm{~h} .{ }^{1} \mathrm{H}$ NMR analysis of the crude reaction mixture indicated the ratio of regioisomers (b/l) to be $92 / 8$. The mixture was purified by flash column chromatography on silica gel (hexanes/ether $10 / 1$ to $5 / 1)$ to give the title compound $(0.143 \mathrm{~g}, 71 \%)$. HPLC analysis indicated that the enantiomeric excess of the product was 98\% [Diacel CHIRALCEL OD-H $(0.46 \mathrm{~cm} \times 25 \mathrm{~cm})$; hexanes $/ 2$-propanol $=99.0 / 1.0 ;$ flow rate $=0.5 \mathrm{~mL} / \mathrm{min}$; detection wavelength $=230 \mathrm{~nm}$; TR $=10.13$ (major), 11.01 (minor) min]. $[\alpha]_{\mathrm{D}}^{25}=+13.6$ (c 1.33, $\mathrm{CHCl}_{3}$ ). ${ }^{1} \mathrm{H}$ NMR $\left(500.13 \mathrm{MHz}, \mathrm{CDCl}_{3}\right) \delta=7.15(\mathrm{dd}, J=5.06,1.4 \mathrm{~Hz}, 1 \mathrm{H}), 6.96-6.90(\mathrm{~m}, 2 \mathrm{H}), 5.77$ (ddd, $J=17.4,10.0,6.9 \mathrm{~Hz}, 1 \mathrm{H}), 5.63$ (dq, $J=12.3,6.7,1 \mathrm{H}), 5.40$ (ddq, $J=15.3,7.5,1.6 \mathrm{~Hz}$, 1H), $5.17(\mathrm{dt}, J=17.4,1.3 \mathrm{~Hz}, 1 \mathrm{H}), 5.10(\mathrm{dm}, J=9.74 \mathrm{~Hz}, 1 \mathrm{H}), 3.94$ (s, 2H), 3.67 (t, $J=7.2 \mathrm{~Hz}$, $1 \mathrm{H}), 1.71(\mathrm{dd}, J=6.7,1.3 \mathrm{~Hz}, 3 \mathrm{H}), 1.58-1.41$ (br s, $1 \mathrm{H}) .{ }^{13} \mathrm{C} \mathrm{NMR}\left(125.7 \mathrm{MHz}, \mathrm{CDCl}_{3}\right) \delta=$ 144.2, 139.9, 132.2, 127.3, 126.6, 124.8, 124.2, 115.5, 62.5, 45.4, 17.8. Anal. Calcd. for $\mathrm{C}_{11} \mathrm{H}_{15} \mathrm{NS}$ : C, 68.35; H, 7.82; N, 7.25; S, 16.59. Found: C, 68.08; H, 7.53; N, 7.31.

(E)- $\mathrm{N}$-(hexa-1,4-dien-3-yl)-4-(methylthio)benzenamine. The general procedure of method B was followed with methyl $(2 E, 4 E)$-hexa-2,4-dienylcarbonate $(0.153 \mathrm{~g}, 0.98 \mathrm{mmol})$, 4aminothioanisole $(0.146 \mathrm{~mL}, 1.50 \mathrm{mmol}),[\operatorname{Ir}(\mathrm{COD}) \mathrm{Cl}]_{2}(6.7 \mathrm{mg}, 0.010 \mathrm{mmol}), \mathbf{L 2}(12.7 \mathrm{mg}$, $0.02 \mathrm{mmol})$ and DABCO $(10 \mathrm{mg}, 0.09 \mathrm{mmol})$. The reaction was conducted at room temperature for $2 \mathrm{~h}$. GC analysis of the crude reaction mixture indicated the ratio of regioisomers (b/l) to be 99/1. The mixture was purified by flash column chromatography on silica gel (hexanes/ether $10 / 1$ to $5 / 1)$ to give the title compound $(0.199 \mathrm{~g}, 93 \%)$. HPLC analysis indicated that the enantiomeric excess of the product was 98\% [Diacel CHIRALCEL OD-H $(0.46 \mathrm{~cm} \times 25 \mathrm{~cm})$; hexanes $/ 2$-propanol $=99.5 / 0.5$; flow rate $=0.5 \mathrm{~mL} / \mathrm{min}$; detection wavelength $=254 \mathrm{~nm} ; \mathrm{TR}=$ 15.3 (major), 16.0 (minor) $\min ] .[\alpha]_{\mathrm{D}}{ }^{25}=+40.4$ (c $\left.0.935, \mathrm{CHCl}_{3}\right) .{ }^{1} \mathrm{H} \mathrm{NMR}(500.13 \mathrm{MHz}$, $\left.\mathrm{CDCl}_{3}\right) \delta=7.19(\mathrm{~d}, J=8.8 \mathrm{~Hz}, 2 \mathrm{H}), 6.57(\mathrm{~d}, J=8.4 \mathrm{~Hz}, 2 \mathrm{H}), 5.85(\mathrm{ddd}, J=17.4,10.4,5.7 \mathrm{~Hz}$, 
1H), $5.71(\mathrm{dqd}, J=15.5,6.5,1.1 \mathrm{~Hz}, 1 \mathrm{H}), 5.48$ (ddq, $J=15.5,6.3,1.6 \mathrm{~Hz}, 1 \mathrm{H}), 5.24(\mathrm{dt}, J=$ 17.4, $1.3 \mathrm{~Hz}, 1 \mathrm{H}), 5.16$ (dt, $J=10.1,1.3 \mathrm{~Hz}, 1 \mathrm{H}), 4.33$ (t, $J=6.1 \mathrm{~Hz}, 1 \mathrm{H}), 4.10-3.80$ (br s, 1H), $2.40(\mathrm{~s}, 3 \mathrm{H}), 1.72(\mathrm{dm}, J=6.6 \mathrm{~Hz}, 3 \mathrm{H}) .{ }^{13} \mathrm{C} \mathrm{NMR}\left(125.7 \mathrm{MHz}, \mathrm{CDCl}_{3}\right) \delta=145.9,138.5,131.2$, 130.7, 127.6, 124.4, 115.5, 114.3, 57.7, 19.1, 17.8. Anal. Calcd. for $\mathrm{C}_{13} \mathrm{H}_{17} \mathrm{NS}$ : C, 71.18; H, 7.81; N, 6.39; S, 14.62. Found: C, 71.15; H, 7.67; N, 6.23.

N-Phenyl-1-vinyl-2-butenylamine. ${ }^{4}$ The general procedure of method B was followed with methyl $(2 E, 4 E)$-hexa-2,4-dienylcarbonate $(0.136,0.87 \mathrm{mmol})$, aniline $(0.116 \mathrm{~mL}, 1.27 \mathrm{mmol})$, $[\operatorname{Ir}(\mathrm{COD}) \mathrm{Cl}]_{2}(6.7 \mathrm{mg}, 0.010 \mathrm{mmol}), \mathbf{L 2}(12.7 \mathrm{mg}, 0.02 \mathrm{mmol})$ and DABCO (10 mg, $\left.0.09 \mathrm{mmol}\right)$. The reaction was conducted at room temperature for $3 \mathrm{~h} .{ }^{1} \mathrm{H}$ NMR analysis of the crude reaction mixture indicated the ratio of regioisomers (b/l) to be 97/3. The mixture was purified by flash column chromatography on silica gel (hexanes/ether $10 / 1$ to $5 / 1$ ) to give the title compound $(0.122 \mathrm{~g}, 81 \%)$. HPLC analysis indicated that the enantiomeric excess of the product was $95 \%$ [Diacel CHIRALCEL OD-H $(0.46 \mathrm{~cm}$ x $25 \mathrm{~cm})$; hexanes/2-propanol = 98.0/2.0; flow rate $=0.5$ $\mathrm{mL} / \mathrm{min}$; detection wavelength $=254 \mathrm{~nm}$; TR $=11.5$ (major), 12.2 (minor) min]. $[\alpha]_{\mathrm{D}}{ }^{25}=-15.2$ $\left(c\right.$ 0.97, $\left.\mathrm{CHCl}_{3}\right) .{ }^{1} \mathrm{H} \mathrm{NMR}\left(400.13 \mathrm{MHz}, \mathrm{CDCl}_{3}\right) \delta=7.11-7.02(\mathrm{~m}, 2 \mathrm{H}), 6.61(\mathrm{td}, J=7.2,0.8 \mathrm{~Hz}$, $1 \mathrm{H}), 6.64(\mathrm{~d}, J=8.8 \mathrm{~Hz}, 2 \mathrm{H}), 5.77(\mathrm{ddd}, J=17.2,10.4,6.0 \mathrm{~Hz}, 1 \mathrm{H}), 5.63(\mathrm{dq}, J=15.2,6.6 \mathrm{~Hz}$, $1 \mathrm{H}), 5.41(\mathrm{ddq}, J=15.2,6.2,0.8 \mathrm{~Hz}, 1 \mathrm{H}), 5.17(\mathrm{dd}, J=17.2,1.4 \mathrm{~Hz}, 1 \mathrm{H}), 5.08$ (dd, $J=10.2$, $1.2 \mathrm{~Hz}, 1 \mathrm{H}), 4.27$ (t, $J=6.4 \mathrm{~Hz}, 1 \mathrm{H}), 3.63(\mathrm{br} \mathrm{s}, 1 \mathrm{H}), 1.63(\mathrm{dd}, J=6.4,0.8 \mathrm{~Hz}, 3 \mathrm{H}) .{ }^{13} \mathrm{C} \mathrm{NMR}$ $\left(100.59 \mathrm{MHz}, \mathrm{CDCl}_{3}\right) \delta=147.2,138.8,131.0,129.0,127.3,117.4,115.3,113.5,57.6,17.8$.

N-benzyl-1-(furan-2-yl)prop-2-en-1-amine. ${ }^{3}$ The general procedure of method A was followed with methyl $(E)-3$-(furan-2-yl)allylcarbonate $(0.211 \mathrm{~g}, 1.16 \mathrm{mmol})$ and benzylamine $(0.140 \mathrm{~mL}$, $1.28 \mathrm{mmol})$. The reaction was conducted at room temperature for $0.5 \mathrm{~h} .{ }^{1} \mathrm{H}$ NMR analysis of the crude reaction mixture indicated the ratio of regioisomers $(b / 1)$ to be $91 / 9$. The mixture was purified by flash column chromatography on silica gel (hexanes/ether 10/1 to 5/1) to give the title compound $(0.200 \mathrm{~g}, 81 \%)$. HPLC analysis indicated that the enantiomeric excess of the product was 98\% [Diacel CHIRALCEL OD-H (0.46 cm x $25 \mathrm{~cm})$; hexanes/2-propanol = 99.9/0.1; flow rate $=0.5 \mathrm{~mL} / \mathrm{min}$; detection wavelength $=230 \mathrm{~nm}$; $\mathrm{TR}=14.6$ (major), 19.2 (minor) $\min ] .[\alpha]_{\mathrm{D}}{ }^{25}=+9.0\left(c 1.065, \mathrm{CHCl}_{3}\right) .{ }^{1} \mathrm{H} \mathrm{NMR}\left(500.13 \mathrm{MHz}, \mathrm{CDCl}_{3}\right) \delta=7.38(\mathrm{~m}, 1 \mathrm{H})$, 7.34-7.31 (m, 4H), 7.28-7.24 (m, 1H), $6.34(\mathrm{dd}, J=3.4,1.7 \mathrm{~Hz}, 1 \mathrm{H}), 6.21(\mathrm{dm}, J=3.4 \mathrm{~Hz}, 1 \mathrm{H})$, 
$5.98(\mathrm{ddd}, J=16.9,10.1,7.3 \mathrm{~Hz}, 1 \mathrm{H}), 5.27(\mathrm{dt}, J=17.1,1.3 \mathrm{~Hz}, 1 \mathrm{H}), 5.24(\mathrm{dt}, J=10.1,1.0 \mathrm{~Hz}$, $1 \mathrm{H}), 4.32(\mathrm{~d}, J=7.3 \mathrm{~Hz}, 1 \mathrm{H}), 3.77(\mathrm{~s}, 2 \mathrm{H}), 2.00$ (br s, $1 \mathrm{H}) .{ }^{13} \mathrm{C} \mathrm{NMR}\left(125.7 \mathrm{MHz}, \mathrm{CDCl}_{3}\right) \delta=$ $155.2,141.8,140.0,137.5,128.4,128.3,127.0,116.9,110.0,106.3,58.3,50.9$.

$N$-benzyl-4-methylpent-1-en-3-amine. The general procedure of method A was followed with (E)-methyl-4-methylpent-2-enylcarbonate $(0.147 \mathrm{~g}, 0.93 \mathrm{mmol})$ and benzylamine $(0.140 \mathrm{~mL}$, $1.28 \mathrm{mmol}) .{ }^{1} \mathrm{H}$ NMR analysis of the crude reaction mixture indicated the ratio of regioisomers (b/l) to be $95 / 5$. The mixture was purified by flash column chromatography on silica gel (hexanes/ether $10 / 1$ to $5 / 1)$ to give the title compound $(0.158 \mathrm{~g}, 90 \%)$. HPLC analysis indicated that the enantiomeric excess of the product was 94\% [Diacel CHIRALCEL OD-H (0.46 cm x 25 $\mathrm{cm}$ ); hexanes $/ 2$-propanol $=99.9 / 0.1$; flow rate $=0.5 \mathrm{~mL} / \mathrm{min}$; detection wavelength $=220 \mathrm{~nm}$; $\mathrm{TR}=6.79$ (major), 5.67 (minor) $\min ] .{ }^{1} \mathrm{H}$ NMR (500.13 MHz, $\left.\mathrm{CDCl}_{3}\right) \delta=7.35-7.20(\mathrm{~m}, 5 \mathrm{H})$, $5.63(\mathrm{ddd}, J=18.7,10.4,8.6 \mathrm{~Hz}, 1 \mathrm{H}), 5.26$ (dd, $J=10.3,2.0 \mathrm{~Hz}, 1 \mathrm{H}), 5.10$ (dqd, $J=17.2,2.0$, $0.8 \mathrm{~Hz}, 1 \mathrm{H}), 3.85(\mathrm{~d}, J=13.5 \mathrm{~Hz}, 1 \mathrm{H}), 3.62(\mathrm{~d}, J=13.5 \mathrm{~Hz}, 1 \mathrm{H}), 2.77$ (dd, $J=8.2,5.7 \mathrm{~Hz}, 1 \mathrm{H})$, $1.70(\mathrm{~m}, 1 \mathrm{H}), 1.30(\mathrm{br} \mathrm{s}, 1 \mathrm{H}), 0.92(\mathrm{~d}, J=6.5 \mathrm{~Hz}, 3 \mathrm{H}), 0.89$ (d, $J=6.5 \mathrm{~Hz}, 3 \mathrm{H}) .{ }^{13} \mathrm{C} \mathrm{NMR}(125.7$ $\left.\mathrm{MHz}, \mathrm{CDCl}_{3}\right) \delta=140.9,139.0,128.3,128.1,126.7,116.9,66.8,51.3,32.2,19.4$, 18.4. Anal. Calcd. for $\mathrm{C}_{13} \mathrm{H}_{19} \mathrm{~N}$ : C, 82.48; H, 10.12; N, 7.40. Found: C, 82.21; H, 10.30; N, 7.50.

$N$-benzyl-1-(4-nitrophenyl)prop-2-en-1-amine. ${ }^{3}$ The general procedure of method A was followed with methyl 4-nitrocinnamylcarbonate $(0.116 \mathrm{~g}, 0.49 \mathrm{mmol})$ and benzylamine $(0.140$ $\mathrm{mL}, 1.28 \mathrm{mmol})$. The reaction was conducted at room temperature for $4 \mathrm{~h}$. ${ }^{1} \mathrm{H}$ NMR analysis of the crude reaction mixture indicated the ratio of regioisomers (b/l) to be $94 / 6$. The mixture was purified by flash column chromatography on silica gel (hexanes/ether 10/1 to 2/1) to give the title compound $(0.108 \mathrm{~g}, 82 \%)$. HPLC analysis indicated that the enantiomeric excess of the product was 96\% [Diacel CHIRALCEL OJ-H $(0.46 \mathrm{~cm} \times 25 \mathrm{~cm})$; hexanes/2-propanol = 95.0/5.0; flow rate $=0.6 \mathrm{~mL} / \mathrm{min}$; detection wavelength $=220 \mathrm{~nm} ; \mathrm{TR}=28.5$ (major), 31.0 (minor) min]. $[\alpha]_{\mathrm{D}}{ }^{25}=-15.2\left(c 0.5, \mathrm{CHCl}_{3}\right) .{ }^{1} \mathrm{H}$ NMR $\left(500 \mathrm{MHz}, \mathrm{CDCl}_{3}\right) \delta=8.20(\mathrm{dm}, J=8.5$ $\mathrm{Hz}, 2 \mathrm{H}), 7.58(\mathrm{dm}, J=8.5 \mathrm{~Hz}, 2 \mathrm{H}), 7.37-7.24(\mathrm{~m}, 6 \mathrm{H}), 5.88$ (ddd, $J=16.9,10.1,7.6 \mathrm{~Hz}, 1 \mathrm{H})$, $5.26(\mathrm{dt}, J=17.1,1.3 \mathrm{~Hz}, 1 \mathrm{H}), 5.20(\mathrm{dm}, J=10.1 \mathrm{~Hz}, 1 \mathrm{H}), 4.35(\mathrm{~d}, J=7.3 \mathrm{~Hz}, 1 \mathrm{H}), 3.50(\mathrm{~d}, J=$ $3.8 \mathrm{~Hz}, 2 \mathrm{H}), 1.70$ (br s, $1 \mathrm{H}) .{ }^{13} \mathrm{C} \mathrm{NMR}\left(125.7 \mathrm{MHz}, \mathrm{CDCl}_{3}\right) \delta=150.3,147.1,139.7,139.5$, 128.5, 128.2, 128.1, 127.2, 123.8, 116.6, 64.6, 51.2. Anal. Calcd. for $\mathrm{C}_{16} \mathrm{H}_{16} \mathrm{~N}_{2} \mathrm{O}_{2}$ : C, 71.62; $\mathrm{H}$, 6.01; N, 10.44; O, 11.93. Found: C, 71.39; H, 6.00; N, 10.24. 
1-Methyl-2-(1-propylallyloxy)benzene. ${ }^{4}$ The general procedure of method A was followed with lithium 2-methylphenoxide $(0.228 \mathrm{~g}, 2.00 \mathrm{mmol})$ and $(E)$-2-hexenyl methylcarbonate $(147 \mathrm{mg}$, $0.93 \mathrm{mmol})$ in THF $(2 \mathrm{~mL})$. The reaction was conducted at room temperature for $2 \mathrm{~h} .{ }^{1} \mathrm{H} \mathrm{NMR}$ analysis of the crude reaction mixture indicated the ratio of regioisomers (b/l) to be $94 / 6$. The mixture was purified by flash column chromatography on silica gel (hexanes/ether 10/1) to give the title compound $(0.158 \mathrm{~g}, 90 \%)$. HPLC analysis indicated that the enantiomeric excess of the product was 98\% [Diacel CHIRALCEL OD-H $(0.46 \mathrm{~cm}$ x $25 \mathrm{~cm})$; hexanes/2-propanol = 99.5/0.5; flow rate $=0.5 \mathrm{~mL} / \mathrm{min}$; detection wavelength $=254 \mathrm{~nm} ; \mathrm{TR}=8.97$ (major), 8.38 (minor) min]. ${ }^{1} \mathrm{H}$ NMR $\left(500.13 \mathrm{MHz}, \mathrm{CDCl}_{3}\right) \delta=7.16(\mathrm{~m}, 2 \mathrm{H}), 6.88(\mathrm{~m}, 2 \mathrm{H}), 5.92(\mathrm{ddd}, J=$ $17.4,10.5,6.1 \mathrm{~Hz}, 1 \mathrm{H}), 5.28(\mathrm{dt}, J=17.4,1.2 \mathrm{~Hz}, 1 \mathrm{H}), 5.22(\mathrm{dt}, J=10.5,1.0 \mathrm{~Hz}, 1 \mathrm{H}), 4.67(\mathrm{dt}, J$ $=6.4,6.1 \mathrm{~Hz}, 1 \mathrm{H}), 2.31(\mathrm{~s}, 3 \mathrm{H}), 1.89-1.83(\mathrm{~m}, 1 \mathrm{H}), 1.76-1.69(\mathrm{~m}, 1 \mathrm{H}), 1.60-1.47(\mathrm{~m}, 2 \mathrm{H}), 1.01$ $(\mathrm{t}, J=7.4 \mathrm{~Hz}, 3 \mathrm{H}) .{ }^{13} \mathrm{C} \mathrm{NMR}\left(125.7 \mathrm{MHz}, \mathrm{CDCl}_{3}\right) \delta=156.5,138.5,130.6,127.4,126.4,120.2$, $115,113.1,78.6,37.9,18.5,16.4,14.0$.

1-Methoxy-4-(1-propylallyloxy)benzene. ${ }^{5}$ The general procedure A was followed with lithium 4-methoxyphenoxide $(0.130 \mathrm{~g}, 1.00 \mathrm{mmol})$ and 2-hexenyl methylcarbonate $(0.085,0.53 \mathrm{mmol})$ in THF $(1 \mathrm{~mL})$. The reaction was conducted at room temperature for $2 \mathrm{~h} .{ }^{1} \mathrm{H} \mathrm{NMR}$ analysis of the mixture indicated the ratio of regioisomers (b/l) to be $95 / 5$. The mixture was purified by flash column chromatography on silica gel (hexanes/ether 10/1) to give the title compound (0.104 g, 94\%). HPLC analysis indicated that the enantiomeric excess of the product was 97\% [Diacel CHIRALCEL OD-H $(0.46 \mathrm{~cm}$ x $25 \mathrm{~cm})$; hexanes $/ 2$-propanol $=99.5 / 0.5$; flow rate $=0.6 \mathrm{~mL} / \mathrm{min}$; detection wavelength $=254 \mathrm{~nm}$; TR $=18.4$ (major), 20.7 (minor) $\min$ ]. ${ }^{1} \mathrm{H}$ NMR $(500.13 \mathrm{MHz}$, $\left.\mathrm{CDCl}_{3}\right) \delta=6.88(\mathrm{~d}, J=9.3 \mathrm{~Hz}, 2 \mathrm{H}), 6.84(\mathrm{~d}, J=9.2 \mathrm{~Hz}, 2 \mathrm{H}), 5.87(\mathrm{ddd}, J=17.3,10.6,6.3 \mathrm{~Hz}$, $1 \mathrm{H}), 5.25(\mathrm{~d}, J=17.3 \mathrm{~Hz}, 1 \mathrm{H}), 5.21(\mathrm{~d}, J=10.6 \mathrm{~Hz}, 1 \mathrm{H}), 4.51$ (q, $J=6.4 \mathrm{~Hz}, 1 \mathrm{H}), 3.80(\mathrm{~s}, 3 \mathrm{H})$, $1.81(\mathrm{~m}, 1 \mathrm{H}), 1.66(\mathrm{~m}, 1 \mathrm{H}), 1.56-1.46(\mathrm{~m} .2 \mathrm{H}), 1.00(\mathrm{t}, J=7.3 \mathrm{~Hz}, 3 \mathrm{H}) .{ }^{13} \mathrm{C} \mathrm{NMR}(125.7 \mathrm{MHz}$, $\left.\mathrm{CDCl}_{3}\right) \delta=153.8,152.5,138.5,117.3,116.2,114.4,79.9,55.6,37.7,18.5,13.9$. 


\section{Allylic Alkylation conducted with $0, O^{\prime}-(R)-\left(1,1^{\prime}-\right.$ Dinaphthyl-2,2'-diyl)-N,N'-di-( $\left.R, R\right)-$ phenylethylphosphoramidite (L1):}

$N$-[1-(n-Propyl)-2-propenyl]benzylamine. The general procedure of method A was followed with the 2-hexenyl methylcarbonate $(0.143 \mathrm{~g}, 0.90 \mathrm{mmol})$ and benzylamine $(0.140 \mathrm{~mL}, 1.28$ mmol). The reaction was conducted at room temperature for $3 \mathrm{~h} .{ }^{1} \mathrm{H}$ NMR analysis of the crude reaction mixture indicated the ratio of regioisomers (b/l) to be $94 / 6$. The mixture was purified by flash column chromatography on silica gel (hexanes/ether 10:1 to 5:1) to give the title compound $(0.108 \mathrm{~g}, 58 \%)$. HPLC analysis indicated that the enantiomeric excess of the product was $95 \%$ [Diacel CHIRALCEL OD-H $(0.46 \mathrm{~cm} \times 25 \mathrm{~cm})$; hexanes $/ 2$-propanol $=98 / 2$; flow rate $=0.5$ $\mathrm{mL} / \mathrm{min}$; detection wavelength $=230 \mathrm{~nm}$; TR $=11.3$ (major), 9.12 (minor) $\mathrm{min}]$.

$N$-Phenyl-1-n-propyl-2-propenylamine. The general procedure A was followed with the 2hexenyl methylcarbonate $(0.149 \mathrm{~g}, 0.94 \mathrm{mmol})$ and aniline $(109 \mathrm{~mL}, 1.20 \mathrm{mmol}) .{ }^{1} \mathrm{H}$ NMR analysis of the residue crude mixture indicated that the ratio of regioisomers (b/l) was 92/8. The crude reaction mixture was purified by flash column chromatography on silica gel (hexanes/ether $20: 1)$ to give desired product $(0.161 \mathrm{~g}, 93 \%)$ as a colorless liquid. HPLC analysis indicated that the enantiomeric excess of the product was 92\% [Diacel CHIRALCEL OD-H $(0.46 \mathrm{~cm} \times 25 \mathrm{~cm})$; hexanes $/ 2$-propanol $=99.95 / 0.05$; flow rate $=0.5 \mathrm{~mL} / \mathrm{min}$; detection wavelength $=254 \mathrm{~nm} ; \mathrm{TR}=$ 14.3 (major), 18.3 (minor) min].

$\mathrm{N}$-(furan-2-ylmethyl)hex-1-en-3-amine. The general procedure of method A was followed with the 2-hexenyl methylcarbonate $(0.149 \mathrm{~g}, 0.94 \mathrm{mmol})$ and furfurylamine $(0.111 \mathrm{~mL}, 1.20 \mathrm{mmol})$. The reaction was conducted at room temperature for $3 \mathrm{~h} .{ }^{1} \mathrm{H}$ NMR analysis of the crude reaction mixture indicated the ratio of regioisomers (b/l) to be $92 / 8$. The mixture was purified by flash column chromatography on silica gel (hexanes/ether $10 / 1$ to $5 / 1$ ) to give the title compound $(0.107 \mathrm{~g}, 69 \%)$. HPLC analysis indicated that the enantiomeric excess of the product was $94 \%$ [Diacel CHIRALCEL OD-H $(0.46 \mathrm{~cm} \times 25 \mathrm{~cm})$; hexanes $/ 2$-propanol $=99.0 / 1.0$; flow rate $=0.5$ $\mathrm{mL} / \mathrm{min}$; detection wavelength $=254 \mathrm{~nm} ; \mathrm{TR}=10.6$ (major), 7.3 (minor) $\mathrm{min}$ ]. 
$N$-(thiophen-2-ylmethyl)hex-1-en-3-amine. The general procedure of method A was followed with 2-hexenyl methylcarbonate $(0.150 \mathrm{~g}, 0.95 \mathrm{mmol})$ and 2-thiophenemethanamine $(0.138 \mathrm{~mL}, 1.30 \mathrm{mmol})$. The reaction was conducted at room temperature for $3 \mathrm{~h} .{ }^{1} \mathrm{H}$ NMR analysis of the crude reaction mixture indicated the ratio of regioisomers (b/l) to be $91 / 9$. The mixture was purified by flash column chromatography on silica gel (hexanes/ether 10/1 to 5/1) to give the title compound $(0.130 \mathrm{~g}, 76 \%)$. HPLC analysis indicated that the enantiomeric excess of the product was 94\% [Diacel CHIRALCEL OD-H $(0.46 \mathrm{~cm}$ x $25 \mathrm{~cm})$; hexanes/2-propanol= 99.0/1.0; flow rate $=0.5 \mathrm{~mL} / \mathrm{min}$; detection wavelength $=230 \mathrm{~nm} ; \mathrm{TR}=11.4$ (major), 10.3 (minor) $\min ]$.

$N$-(hex-1-en-3-yl)-4-(methylthio)benzenamine. The general procedure of method A was followed with 2-hexenyl methylcarbonate $(0.153 \mathrm{~g}, 0.96 \mathrm{mmol})$ and 4-aminothioanisole $(0.146$ $\mathrm{mL}, 1.20 \mathrm{mmol})$. The reaction was conducted at room temperature for $3 \mathrm{~h}$. ${ }^{1} \mathrm{H}$ NMR analysis of the crude reaction mixture indicated the ratio of regioisomers (b/1) to be $99 / 1$. The mixture was purified by flash column chromatography on silica gel (hexanes/ether 10/1) to give the title compound (0.195 g, 94\%). HPLC analysis indicated that the enantiomeric excess of the product was 92\% [Diacel CHIRALCEL OD-H (0.46 cm x $25 \mathrm{~cm})$; hexanes/2-propanol = 99.5/0.5; flow rate $=0.5 \mathrm{~mL} / \mathrm{min}$; detection wavelength $=230 \mathrm{~nm}$; TR $=18.6$ (major), 17.9 (minor) min].

(E)-N-(furan-2-ylmethyl)hexa-1,4-dien-3-amine. The general procedure of method B was followed with $(2 E, 4 E)$-hexa-2,4-dienylmethylcarbonate $(0.145 \mathrm{~g}, 0.92 \mathrm{mmol})$, furfurylamine (0.140 mL, $1.58 \mathrm{mmol}),[\operatorname{Ir}(\mathrm{COD}) \mathrm{Cl}]_{2}(6.7 \mathrm{mg}, 0.010 \mathrm{mmol}), \mathrm{L} 1(12.7 \mathrm{mg}, 0.02 \mathrm{mmol})$ and DABCO $(10 \mathrm{mg}, 0.09 \mathrm{mmol})$. The reaction was conducted at room temperature for $18 \mathrm{~h} .{ }^{1} \mathrm{H}$ NMR analysis of the crude reaction mixture indicated the ratio of regioisomers (b/l) to be 80/20. The mixture was purified by flash column chromatography on silica gel (hexanes/ether 10/1 to $5 / 1)$ to give the title compound $(0.079 \mathrm{~g}, 48 \%)$. HPLC analysis indicated that the enantiomeric excess of the product was 96\% [Diacel CHIRALCEL OD-H $(0.46 \mathrm{~cm}$ x $25 \mathrm{~cm})$; hexanes/2propanol $=99.9 / 0.1 ;$ flow rate $=0.5 \mathrm{~mL} / \mathrm{min}$; detection wavelength $=230 \mathrm{~nm} ; \mathrm{TR}=8.72$ (major), 7.85 (minor) $\mathrm{min}]$. 
(E)-N-(thiophen-2-ylmethyl)hexa-1,4-dien-3-amine. The general procedure of method B was followed with $(2 E, 4 E)$-hexa-2,4-dienylmethylcarbonate $(0.157 \mathrm{~g}, 1.00 \mathrm{mmol})$, thiophenemethanamine $(0.138 \mathrm{~mL}, 1.34 \mathrm{mmol}),[\operatorname{Ir}(\mathrm{COD}) \mathrm{Cl}]_{2}(6.7 \mathrm{mg}, 0.010 \mathrm{mmol}), \mathrm{L} 1(12.7$ $\mathrm{mg}, 0.02 \mathrm{mmol})$ and DABCO $(10 \mathrm{mg}, 0.09 \mathrm{mmol})$. The reaction was conducted at room temperature for $18 \mathrm{~h} .{ }^{1} \mathrm{H}$ NMR analysis of the crude reaction mixture indicated the ratio of regioisomers $(\mathrm{b} / \mathrm{l})$ to be $82 / 18$. The mixture was purified by flash column chromatography on silica gel (hexanes/ether 10/1 to 5/1) to give the title compound (0.094 g, 49\%). HPLC analysis indicated that the enantiomeric excess of the product was 92\% [Diacel CHIRALCEL OD-H $(0.46 \mathrm{~cm} \times 25 \mathrm{~cm})$; hexanes $/ 2$-propanol $=99.0 / 1.0$; flow rate $=0.5 \mathrm{~mL} / \mathrm{min}$; detection wavelength $=230 \mathrm{~nm} ; \mathrm{TR}=11.04$ (major), 10.12 (minor) $\min ]$.

(E)- $N$-(hexa-1,4-dien-3-yl)-4-(methylthio)benzenamine. The general procedure of method B was followed with $(2 E, 4 E)$-hexa-2,4-dienylmethylcarbonate $(0.153 \mathrm{~g}, 0.97 \mathrm{mmol})$, 4aminothioanisole $(0.146 \mathrm{~mL}, 1.50 \mathrm{mmol}),[\operatorname{Ir}(\mathrm{COD}) \mathrm{Cl}]_{2}(6.7 \mathrm{mg}, 0.010 \mathrm{mmol}), \mathrm{L} 1$ (12.7 mg, $0.02 \mathrm{mmol})$ and DABCO $(10 \mathrm{mg}, 0.09 \mathrm{mmol})$. The reaction was conducted at room temperature for $18 \mathrm{~h}$. ${ }^{1} \mathrm{H}$ NMR analysis of the crude reaction mixture indicated the ratio of regioisomers (b/l) to be 93/7. The mixture was purified by flash column chromatography on silica gel (hexanes/ether 10/1) to give the title compound (0.161 g, 73\%). HPLC analysis indicated that the enantiomeric excess of the product was 93\% [Diacel CHIRALCEL OD-H $(0.46 \mathrm{~cm} \times 25 \mathrm{~cm})$; hexanes $/ 2$-propanol $=99.5 / 0.5$; flow rate $=0.5 \mathrm{~mL} / \mathrm{min}$; detection wavelength $=254 \mathrm{~nm} ; \mathrm{TR}=$ 16.0 (major), 15.3 (minor) $\min ]$.

$N$-Phenyl-1-vinyl-2-butenylamine. The general procedure of method B was followed with (2E, 4E)-hexa-2,4-dienylmethylcarbonate $(0.144 \mathrm{~g}, 0.91 \mathrm{mmol})$, aniline $(0.116 \mathrm{~mL}, 1.27 \mathrm{mmol})$, $[\operatorname{Ir}(\mathrm{COD}) \mathrm{Cl}]_{2}(6.7 \mathrm{mg}, 0.010 \mathrm{mmol}), \mathrm{L} 1(10.7 \mathrm{mg}, 0.02 \mathrm{mmol})$ and DABCO (10 mg, $\left.0.09 \mathrm{mmol}\right)$ . ${ }^{1} \mathrm{H}$ NMR analysis of the crude reaction mixture indicated the ratio of regioisomers $(\mathrm{b} / \mathrm{l})$ to be 71/29. The mixture was purified by flash column chromatography on silica gel (hexanes/ether $10 / 1)$ to give the title compound $(0.124 \mathrm{~g}, 78 \%)$. HPLC analysis indicated that the enantiomeric excess of the product was 97\% [Diacel CHIRALCEL OD-H $(0.46 \mathrm{~cm}$ x $25 \mathrm{~cm})$; hexanes/2propanol $=98.0 / 2.0 ;$ flow rate $=0.5 \mathrm{~mL} / \mathrm{min} ;$ detection wavelength $=254 \mathrm{~nm} ; \mathrm{TR}=12.2$ (major), 11.5 (minor) $\min ]$. 
N-benzyl-1-(furan-2-yl)prop-2-en-1-amine. The general procedure of method A was followed with $(E)$-3-(furan-2-yl)allylmethylcarbonate $(0.174 \mathrm{~g}, 0.96 \mathrm{mmol})$, benzylamine $(0.140 \mathrm{~mL}, 1.28$ mmol). The reaction was conducted at room temperature for $1 \mathrm{~h} .{ }^{1} \mathrm{H}$ NMR analysis of the crude reaction mixture indicated the ratio of regioisomers $(\mathrm{b} / \mathrm{l})$ to be $84 / 16$. The mixture was purified by flash column chromatography on silica gel (hexanes/ether $10 / 1$ to $5 / 1$ ) to give the title compound $(0.083 \mathrm{~g}, 41 \%)$. HPLC analysis indicated that the enantiomeric excess of the product was 95\% [Diacel CHIRALCEL OD-H (0.46 cm x $25 \mathrm{~cm})$; hexanes/2-propanol = 99.9/0.1; flow rate $=0.5 \mathrm{~mL} / \mathrm{min}$; detection wavelength $=230 \mathrm{~nm} ; \mathrm{TR}=15.2$ (major), 20.8 (minor) min].

$N$-benzyl-4-methylpent-1-en-3-amine. The general procedure of method A was followed with (E)-methyl-4-methylpent-2-enylcarbonate $(0.150 \mathrm{~g}, 0.95 \mathrm{mmol})$, benzylamine $(0.140 \mathrm{~mL}, 1.28$ mmol). The reaction was conducted at room temperature for $14 \mathrm{~h} .{ }^{1} \mathrm{H}$ NMR analysis of the crude reaction mixture indicated the ratio of regioisomers $(b / 1)$ to be $92 / 8$. The mixture was purified by flash column chromatography on silica gel (hexanes/ether 10/1 to 5/1) to give the title compound $(0.119 \mathrm{~g}, 66 \%)$. HPLC analysis indicated that the enantiomeric excess of the product was $89 \%$ [Diacel CHIRALCEL OD-H $(0.46 \mathrm{~cm}$ x $25 \mathrm{~cm})$; hexanes/2-propanol $=98 / 2$; flow rate $=0.5$ $\mathrm{mL} / \mathrm{min}$; detection wavelength $=220 \mathrm{~nm} ; \mathrm{TR}=6.81$ (major), 5.68 (minor) $\min$ ].

N-benzyl-1-(4-nitrophenyl)prop-2-en-1-amine. The general procedure of method A was followed with 4-nitrocinnamylmethylcarbonate $(0.116 \mathrm{~g}, 0.49 \mathrm{mmol})$, benzylamine $(0.140 \mathrm{~mL}$, $1.28 \mathrm{mmol})$. The reaction was conducted at room temperature for $14 \mathrm{~h} .{ }^{1} \mathrm{H}$ NMR analysis of the crude reaction mixture indicated the ratio of regioisomers $(b / 1)$ to be $83 / 17$. The mixture was purified by flash column chromatography on silica gel (hexanes/ether 10/1 to 5/1) to give the title compound $(0.098 \mathrm{~g}, 75 \%)$. HPLC analysis indicated that the enantiomeric excess of the product was 96\% [Diacel CHIRALCEL OJH (0.46 cm x $25 \mathrm{~cm})$; hexanes/2-propanol = 99.9/0.1; flow rate $=0.5 \mathrm{~mL} / \mathrm{min}$; detection wavelength $=254 \mathrm{~nm}$; $\mathrm{TR}=30.4$ (major), 28.8 (minor) $\min$ ]. 
1-Methyl-2-(1-propylallyloxy)benzene. The general procedure of method A was followed with lithium 2-methylphenoxide (114 mg, $1.0 \mathrm{mmol})$ and 2-hexenyl methylcarbonate $(70 \mathrm{mg}, 0.48$ mmol) in THF (1 mL). The reaction was conducted at RT for $14 \mathrm{~h} .{ }^{1} \mathrm{H}$ NMR analysis of the crude reaction mixture indicated the ratio of regioisomers (b/l) to be $92 / 8$. The mixture was purified by flash column chromatography on silica gel (hexanes/ether 10/1) to give the title compound (0.066 g, 79\%). HPLC analysis indicated that the enantiomeric excess of the product was 94\% [Diacel CHIRALCEL OD-H $(0.46 \mathrm{~cm}$ x $25 \mathrm{~cm})$; hexanes/2-propanol = 99.5/0.5; flow rate $=0.5 \mathrm{~mL} / \mathrm{min}$; detection wavelength $=254 \mathrm{~nm} ; \mathrm{TR}=8.38$ (major), 8.96 (minor) $\min ]$.

1-Methoxy-4-(1-propylallyloxy)benzene. The general procedure of method A was followed with lithium 4-methoxyphenoxide (130 mg, $1.0 \mathrm{mmol})$ and 2-hexenyl methylcarbonate $(85 \mathrm{mg}$, $0.53 \mathrm{mmol})$ in THF $(1 \mathrm{~mL})$. The reaction was conducted at RT for $2 \mathrm{~h} .{ }^{1} \mathrm{H}$ NMR analysis of the crude mixture indicated the ratio of regioisomers (b/l) $95 / 5$. The mixture was purified by flash column chromatography on silica gel (hexanes/ether 10/1) to give the title compound $(0.106 \mathrm{~g}$, 95\%). HPLC analysis indicated that the enantiomeric excess of the product was 94\% [Diacel CHIRALCEL OD-H $(0.46 \mathrm{~cm}$ x $25 \mathrm{~cm})$; hexanes $/ 2$-propanol $=99.5 / 0.5$; flow rate $=0.6 \mathrm{~mL} / \mathrm{min}$; detection wavelength $=254 \mathrm{~nm} ; \mathrm{TR}=20.5$ (major), 18.6 (minor) $\min$ ].

\footnotetext{
${ }^{1}$ Alexakis, A.; Rosset, S.; Allamand, J.; March, S.; Guillen, F.; Benhaim, C. Synlett 2001, 1375-1378.

${ }^{2}$ Naasz, R.; Arnold, L. A.; Minnaard, A. J.; Feringa, B. L. Angew. Chem. Int. Ed. 2001, 40, 927.

${ }^{3}$ Ohmura, T.; Hartwig, J. F. J. Am. Chem. Soc. 2002, 124, 15164-15165.

${ }^{4}$ Shu, C.; Leitner, A.; Hartwig, J. F. Angew. Chem. Int. Ed. 2004, 43, 4797-4800.

${ }^{5}$ Lopez, F.; Ohmura, T.; Hartwig, J. F. J. Am. Chem. Soc. 2003, 125, 3426-3427.
} 